\title{
Nonlinear microwave properties of ferroelectric thin films
}

\author{
R. Ott, P. Lahl, and R. Wördenweber ${ }^{\text {a) }}$ \\ Institut für Schichten und Grenzflächen (ISG) and cni-Center of Nanoelectronic Systems for Information \\ Technology, Forschungszentrum Jülich, 52425 Jülich, Germany
}

(Received 4 August 2003; accepted 30 March 2004; published online 6 May 2004)

\begin{abstract}
The nonlinear microwave properties of ferroelectric $\mathrm{SrTiO}_{3}$ thin films are characterized via complex analysis of the intermodulation distortion (IMD) signals up to high microwave power. The measurements reveal an unusual dependence of the IMD signals on the input power, which indicates the presence of two different nonlinear properties being responsible for the generation of IMD, i.e., the nonlinear permittivity and conductivity at low and high rf power levels, respectively. The IMD signal strength cannot be explained in terms of the classical description based on a Taylor expansion of the nonlinear parameter. In contrast, simulations of the frequency spectra using more appropriate descriptions of two nonlinear parameters yield an excellent agreement between theory and experiment, and, thus, demonstrate that the IMD experiments together with the simulation might provide further insight into the mechanism of nonlinear behavior of these materials. (c) 2004 American Institute of Physics. [DOI: 10.1063/1.1751223]
\end{abstract}

The rapid development of microwave applications in a broad variety of technologies (e.g., communication, radar, anti-collision systems, etc.) has triggered a strong demand for frequency-tunable resonators and filters operating in the $\mathrm{GHz}$ regime. Thin films of ferroelectrics provide high relative permittivity $\varepsilon$, which can be modified by a dc electric field. Therefore, these materials can be utilized in tunable capacitors for electronic devices. In contrast to bulk dielectrics, they can be integrated into small complex thin film devices and, for instance, be used in combination with perovskite superconductors of extremely low rf losses. However, up to the present a few problems inherent to these materials have to be solved. One of those is given by the nonlinear behavior of $\varepsilon$ at high levels of microwave power. The latter effect has not attracted too much interest up to now. However, in the case of device integration and miniaturization, the tunable elements can be exposed to large rf power and, thus, nonlinear effects might become important.

In this letter we will focus on the nonlinear dielectric properties of ferroelectric thin films. The nonlinear dielectric properties are analyzed by measurements of third (and higher) order intermodulation distortion (IMD) signals on $\mathrm{SrTiO}_{3}$ (STO) thin film varactors. The experimental results are compared with theoretical predictions based on Taylor expansions of the nonlinear permittivity and numerical simulations.

The STO films (thickness of $400 \mathrm{~nm}$ ) are grown on $\mathrm{CeO}_{2}$-buffered $r$-cut sapphire via on-axis magnetron rf sputtering technique. The structural orientation of STO strongly depends on gas pressure and substrate temperature during deposition. At low process pressure and temperature, STO growths predominantly (110) oriented, at higher temperature $T_{H}>840^{\circ} \mathrm{C}$ additionally (111)-oriented STO is observed and leads to a relaxation of the film. The relative permittivity and losses of the films are shown in Fig. 1; the temperature dependence and absolute values are comparable to literature

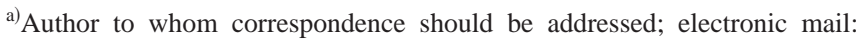
r.woerdenweber@fz-juelich.de
}

values. ${ }^{1-3}$ The fractional tunability $\left[\varepsilon(0 \mathrm{~V})-\varepsilon\left(U_{\mathrm{dc}}\right)\right] /$ $\varepsilon(0 \mathrm{~V})$ scales with $\varepsilon$ and ranges from $20 \%$ to $50 \%$ for electrical fields $E_{\mathrm{dc}}=50 \mathrm{~V} / \mu \mathrm{m}$. An extensive discussion of the structural and dielectric properties and their correlation will be given in a forthcoming article.

The nonlinear behavior of the STO films is determined by IMD measurements. Two fundamental rf signals with slightly different frequencies (here: $f_{1}=1.94 \mathrm{GHz}, f_{2}$ $=1.96 \mathrm{GHz}$ ) automatically generate higher harmonic signals and IMD signals $P_{ \pm i}(i=3,5,7, \ldots)$ at frequencies $f_{n, m}=n f_{1}$ $+m f_{2}$. The most prominent signals are the third order IMD signals $P_{ \pm 3}$ at $2 f_{1}-f_{2}$ and $2 f_{2}-f_{1}$, respectively, which are also closest to the fundamental signals (see Fig. 2).

Generally, the recorded IMD signals are relatively small. In the worst case, they exceed the noise floor at rf inputpowers $P_{\text {in }}>-7 \mathrm{dBm}$, differences of the signal levels $\Delta P$ $=P_{\text {in }}-P_{\text {IMD }}<40 \mathrm{~dB}$ are obtained at $P_{\text {in }} \leqslant 20 \mathrm{dBm}$. This seems to be sufficient for most applications. However, there is a clear tendency that the IMD power level increases with increasing $\varepsilon$ and tunability. Thus, in the case of further improvement of $\varepsilon$ and miniaturization of the STO elements, IMD signals might become important.

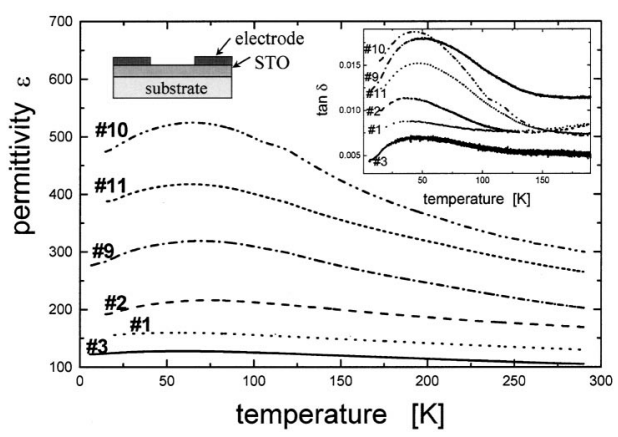

FIG. 1. Temperature dependence of permittivity and loss tangent as a function of temperature our STO films. The STO films are $400 \mathrm{~nm}$ thick and deposited via magnetron-sputter technology on $\mathrm{CeO}_{2}$ buffered sapphire at different heater temperatures $\left(1: T_{H}=650^{\circ} \mathrm{C}, 2: 695^{\circ} \mathrm{C}, 3: 728{ }^{\circ} \mathrm{C}, 9\right.$ : $858^{\circ} \mathrm{C}, 10: 861^{\circ} \mathrm{C}, 11: 867^{\circ} \mathrm{C}$ ). The sketch shows a schematic layout of the planar capacitor that was used for all measurements. 

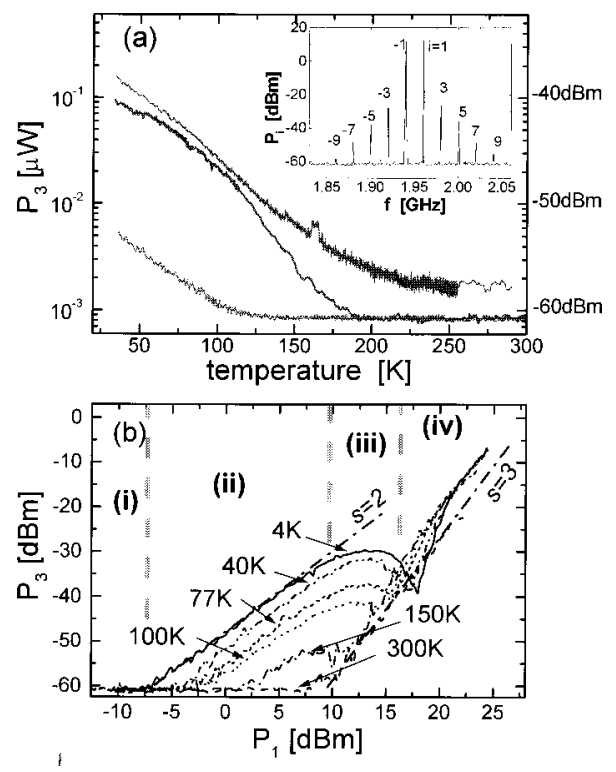

FIG. 2. (a) Temperature dependence of the third order IMD signal of different STO films measured at $P_{\text {in }}=17 \mathrm{dBm}(50 \mathrm{~mW})$ on planar capacitors (2 $\mu \mathrm{m}$ gap width, see sketch Fig. 1), the noise level of the experiment is $\sim 60$ $\mathrm{dBm}$. The inset shows a typical spectrum of IMD measurements with IMD signals up to the ninth order. (b) third order IMD signal as a function of the fundamental signal for sample 10 at different temperatures. The roman numbers mark different regimes, the dashed lines indicate different power dependences with slopes 2 and 3 , respectively.

More amazing is the power dependence of the IMD signal. All IMD signals show a similar behavior as shown in Fig. 2(b) for the third order signal. At high temperature the usual linear increase with slope 3 (third order power dependence) is observed. At lower temperatures (i) a quadratic increase of the third order IMD signal with input power is observed and (ii) a second mechanism becomes visible, that is separated from the other mechanism by a clear dip in the IMD curve. The quadratic increase has been observed for STO varactors in low-power IMD experiment. ${ }^{4}$ It has to be caused by the presence of two nonlinear propertiespermittivity $\varepsilon(E)$ and conductivity $G(E)$ —which will be demonstrated by the two following experiments.

Nonlinear relative permittivity $\varepsilon(E)$ : The nonlinearity of $\varepsilon$ is a consequence of the tunability of STO (see inset of Fig. 3 ). A test of this mechanism regarding IMD generation is provided by IMD measurements with additional dc electrical field [see Fig. 3(a)]. With increasing dc field, the IMD signal is gradually suppressed in regime (ii). At $E_{\mathrm{dc}}=25 \mathrm{~V} / \mu \mathrm{m}$ (50 $\mathrm{V})$ the first mechanism is removed and the measurement resembles the data of $U_{\mathrm{dc}}=0$ at $300 \mathrm{~K}$ [Fig. 2(b)]. Thus, it seems that the first increase (and the dip) in the IMD power can be ascribed to the nonlinearity of $\varepsilon$.

Nonlinear conductivity $G(E)$ : In a second experiment the dc electric conductivity of the STO varactor is analyzed simultaneously with the IMD signal. There seems to be a clear correlation between the onset of conductivity and the second IMD mechanism [Fig. 3(b)]. Similar results are obtained for other temperatures.

Finally, we compare the experiment with numerical simulation of the IMD signals. Usually IMD signals are theoretically described starting from a Taylor expansion of the nonlinear parameter. ${ }^{5}$ In an attempt to parametrize the nonlinear dependence of the IMD signal of ferroelectric maDownloaded 21 Dec 2006 to 134.94.122.39. Redistribution subjec
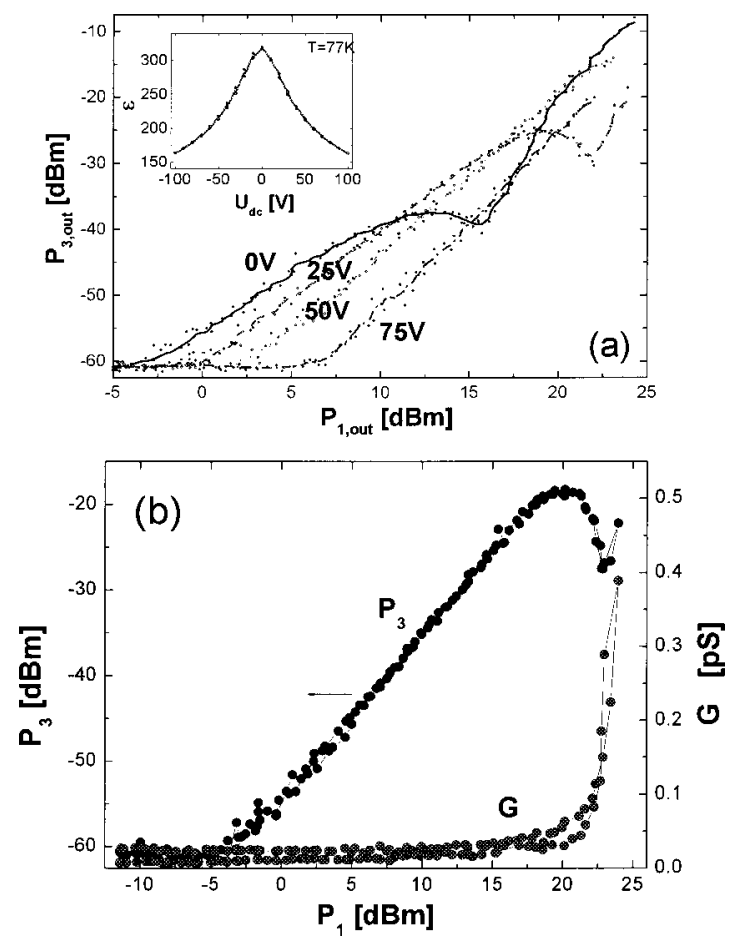

FIG. 3. (a) Third order IMD signal level as a function of the fundamental signal level for different dc bias voltages at $4 \mathrm{~K}$. The inset shows a typical dc bias dependence of $\varepsilon$ ( $2 \mu \mathrm{m}$ gap width). (b) Comparison of the dc conductivity and $P_{3}$ as a function of off power level $P_{1}$ at $4 \mathrm{~K}$.

terial, the nonlinear dependence of the electric displacement $D(E)$ would be expressed in terms of a power series: ${ }^{6}$

$$
D(E)=\varepsilon_{0} \varepsilon(E) \cdot E=\sum k_{i} E^{i}=k_{1} E+k_{3} E^{3}+k_{5} E^{5} \ldots
$$

Due to symmetry reasons the odd powers of $\varepsilon(E)$ vanish. This approach leads to characteristic power dependencies of the IMD signals, i.e., $P_{ \pm i}$ increases steadily and with slopes $s=i .^{5}$ This is not what we observe in the experiment. Apparently there are a few shortcomings of the model: The Taylor expansion used for analytic solution of the IMD problem is usually considered up to order $3 \leqslant n \leqslant 5$. This only describes the onset of the nonlinearity, whereas we are measuring up to large powers. The nonlinearity of $\varepsilon$ and $G$ is quite complex. For instance, $\varepsilon(E)$ levels off at high power [see inset of Fig. 3(a)] and $G(E)$ shows a sharp onset at high power [Fig. 3(b)]. Generally, it seems to be difficult to analytically describe the effect of more than one nonlinear mechanism at large power (i.e., far from the origin) via a Taylor expansion with terms of up to a restricted order $n$.

Thus, we developed a numerical procedure for the simulation of the IMD signal starting with a more complex field dependence of $\varepsilon$ : (i) two wave functions $E_{i}(t)$ of slightly different frequencies are added, (ii) the nonlinear response of the dielectric material $D(t)=\Sigma k_{i}\left[\left(E_{1}^{i}(t)+E_{2}^{i}(t)\right]\right.$ is calculated and (iii) the spectrum is derived via Fourier transformation of $D(t)$. Using an $\varepsilon(E)$ dependence that resembles the experimental curve [compare insets of Figs. 3(a) and 4] this simulation yields intermodulation signals $P_{ \pm i}$ with $i$ $=3,5,7, \ldots$ at the expected frequencies. The resolution of the simulation is restricted by the number of data points in time space. Above this resolution limit (here: $P_{+i} \approx 0.05$ ), the o AlP license or copyright, see http://apl.aip.org/apI//copyright.jsp 


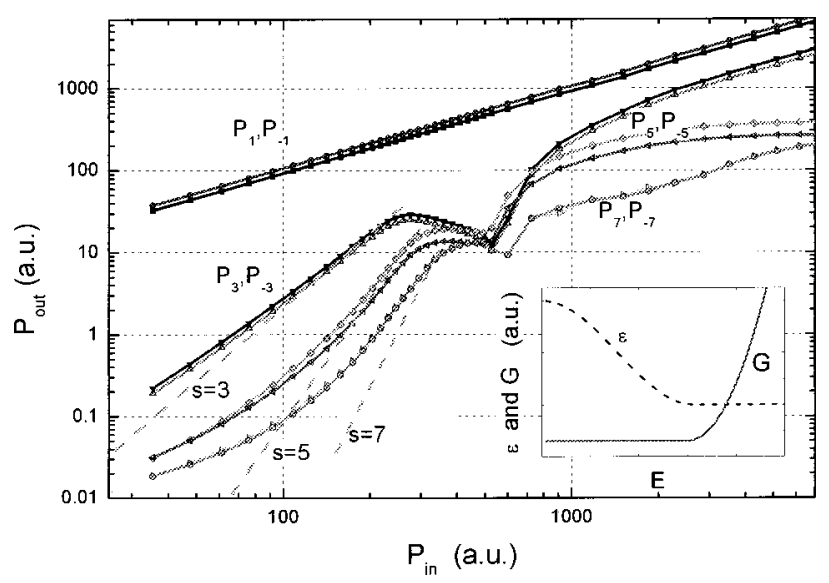

FIG. 4. Simulation of the IMD signal power $P_{i}$ as a function of input power. The nonlinearity of the input parameters $\varepsilon(E)$ and $G(E)$ are sketched in the inset. All parameters are given in arbitrary units, the dashed lines represent the slopes expected for the simulation based on a Taylor expansion of the nonlinear parameters.

IMD signals show a power dependence similar to the experimental data. $P_{ \pm i}$ first increases with slope $s \approx 2$, followed by a leveling off-caused by the leveling off of the $\varepsilon(E)$-dependence- and a decrease with increasing rf input power. Adding the second mechanism (nonlinear conductivity) to the simulation by introducing a second nonlinear description of $D(t)$ with an offset (see inset of Fig. 4) causes a steady increase of the IMD signal level at larger power levels. The slope of this increase is defined by the exact function $D(E)$. Inserting reasonable parameters $k_{i}$ for this mechanism, we can simulate the complete power dependence of the IMD signals.
In conclusion, our measurements of the resulting IMD signals of STO capacities reveal (i) that STO films possess a large power handling capability which, however, decreases with increasing $\varepsilon$, and (ii) that the power dependence of the IMD signals is quite unusual. Two different nonlinear mechanisms are identified, i.e., the nonlinear electric-field dependence of $\varepsilon$ and $G$. A theoretical explanation in terms of a standard Taylor expansion up to order $n<7$ of the nonlinear parameters cannot explain the results. Simulation using more appropriate descriptions of $\varepsilon$ and $G$ up to large $\mathrm{rf}$ power shows an excellent agreement between experimental and simulated data. The simulation procedure might be a useful tool to interpret not understood IMD results in literature, ${ }^{6}$ it might also help to unravel the mechanism responsible for the tunability of ferroelectric thin films.

The authors would like to acknowledge the help of S. Bunte, P. Dymashevski, W. Hofer, E. Hollmann, R. Kutzner, N. Klein, A. Kozyrev, O. and I. Vendik.

${ }^{1}$ M. B. Lee and H. Koinuma, J. Appl. Phys. 81, 2358 (1997); K. Petrov and E. F. Carlson, J. Appl. Phys. 84, 3134 (1997).

${ }^{2}$ O. G. Vendik, E. K. Hollmann, A. B. Kozyrev, and A. M. Prudan, J. Supercond. 12, 325 (1999).

${ }^{3}$ K. Bouzehouane, P. Woodall, B. Marcilhac, A. N. Khodan, D. Crété, E. Jacquet, J. C. Mage, and J. P. Contour, Appl. Phys. Lett. 80, 109 (2002).

${ }^{4}$ B. Kozyrev, T. B. Samoilova, A. A. Golovkov, E. K. Hollmann, D. A. Kalinikos, V. E. Loginov, A. M. Prudan, O. I. Soldatenkov, D. Galt, C. H. Mueller, T. V. Rivkin, and G. A. Koepf, J. Appl. Phys. 84, 3326 (1998).

${ }^{5} \mathrm{M}$. Hein, High-Temperature-Superconductor Thin Films at Microwave Frequencies (Spinger, Berlin, 1999).

${ }^{6}$ N. A. Pertsev, A. G. Zembilgotov, and A. K. Tagantsev, Phys. Rev. Lett. 80, 1988 (1998). 